\title{
SINGLE-FREQUENCY PULSED LASER OSCILLATOR AND SYSTEM FOR LASER-ULTRASONICS
}

A.Blouin, L. Carrion, C. Padioleau, P.Bouchard, J.-P. Monchalin

Industrial Materials Institute, National Research Council Canada, Boucherville, Québec, Canada

\begin{abstract}
We present a new pulsed laser oscillator and system for the optical detection of ultrasound in materials. A single-frequency laser oscillator based on a pulse pumped Nd:YAG rod inside a ring cavity is proposed. The laser delivers single-frequency pulses of $35 \mathrm{~W}$ power. Power of about $1 \mathrm{~kW}$ can be obtained when the second rod of a dual-rod pumping chamber is used as an amplifier. Performance of the system is then investigated with a GaAs photorefractive crystal-based two-wave mixing phase demodulator. In particular, the intensity noise of the laser can be made low enough to allow the detection limit to be set by the shot-noise of the laser. The coherence length of the laser is about $20 \mathrm{~m}$, which makes it a versatile laser-ultrasonic inspection system operated with a two-wave mixing based phase demodulator. A complete compact and affordable system is obtained when the second rod of the pumping chamber is used as a generation laser of ultrasound. Tests of this laser-ultrasonic system on metallic samples are presented. In that case the second rod of the pumping chamber is used as an ultrasound generation laser.
\end{abstract}

Introduction: Laser generation and detection of ultrasound presents numerous advantages for material inspection and characterization over classical piezoelectric-based techniques [1]. In particular, lasers allow probing parts with complex shape in severe conditions, such as high temperature or parts moving at high speed on a production line [2]. A laser-ultrasonic system is typically made of two units, one for generation and another one for detection of ultrasound. The generation unit is essentially a short pulse laser with sufficient power to generate adequately ultrasound in the material. The detection unit typically includes a detection laser, cw or long pulse, and an optical interferometer. The interferometer, working as a phase demodulator, is used to measure the small ultrasonic displacements (typically in the Angstrom or nanometer range) produced at the surface of the tested part by the ultrasonic waves. Since insensitivity to laser speckle caused by surface roughness is preferable for practical use in industry, speckle insensitive phase demodulator schemes based on a confocal Fabry-Perot have been developed more than a decade ago [3]. More recently, adaptive phase demodulator schemes based on two-wave mixing in a photorefractive crystal were developed $[4,5,6]$. In a photorefractive two-wave mixing-based phase demodulator (TWMPD), a pump beam interferes inside the crystal with a signal beam that has acquired a phase modulation after reflection by the inspected part. An interference grating or more generally a hologram, is created and diffracts the pump beam in the direction of the signal beam, thus creating a local oscillator beam which is speckle-adapted to the signal beam. Photorefractive-based adaptive phase demodulators are a good alternative to confocal Fabry-Perot interferometers for ultrasonic detection [5].

In addition to the phase demodulator, the laser-ultrasonic detection unit requires a detection laser. This detection laser should meet stringent conditions regarding its power and noise figure. The detection laser should be sufficiently powerful to get an adequate sensitivity for remote measurement of the small ultrasonic surface displacements on highly scattering and absorbing industrial surfaces. In practice, such a power is obtained with a pulsed system; a pulse duration of about $50 \mu \mathrm{s}$ is usually sufficient to capture several ultrasonic echoes bouncing back and forth in the material. The laser intensity and phase fluctuations should also be sufficiently small so that the dominant noise source is the shot-noise. The spectrum of these fluctuations is usually dominated by the frequency peaks at the relaxation oscillation of the laser and its harmonics. With a proper laser design, the relaxation oscillation frequency is kept outside the ultrasonic frequency range, allowing to reduce this noise source by electronic filtering. Finally, the coherence length of the laser should be also sufficiently large for adequate operation of the demodulator, although this coherence requirement is substantially diminished with two-wave mixing demodulators since the signal and beam paths could be made nearly equal in this case. To fulfill these requirements, current practice is to start from a very stable single-frequency, cw diode-pumped Nd:YAG laser oscillator and to amplify it through a series of flashlamp-pumped or diode-pumped Nd:YAG amplifiers, this approach is commonly named Master-Oscillator Power Amplifier or MOPA. These laser sources can deliver pulses up to a few $\mathrm{kW}$ peak power and of very low intensity and phase noises. The coherence length of these laser sources is several $\mathrm{km}$, which is actually much more than needed. These laser sources include many optical elements (several amplifier stages or multi-passes schemes and Faraday isolators to prevent spurious lasing) and consequently tend to have a high cost, which is a significant impediment for broader use of laser-ultrasonics. 
We present here the results of an effort to make this technology more affordable, particularly by a simplification of the detection laser. Instead of starting from an extremely stable $\mathrm{cw}$ diode-pumped oscillator but relatively low power, we have developed a pulsed oscillator with much higher power and adequate characteristics. Depending upon the application, this pulsed oscillator can be used directly without further amplification. In one version, this pulsed oscillator is followed by an amplifier to provide output power and characteristics which, for laser-ultrasonic measurements, are essentially equivalent to the presently existing $\mathrm{kW}$ units composed of a cW oscillator followed by amplifiers. In another version, the amplifier stage is turned into a Q-switch generation laser, thus providing an even more cost effective system. This pulsed oscillator, which is affected by some intensity and phase noise and has a limited coherence length, is preferably associated with a TWMPD rather than a confocal Fabry-Perot. TWMPDs have the advantage to provide excellent rejection against intensity noise from the laser source by the associated balanced detection scheme. Reduction of the intensity fluctuations of the laser by $30 \mathrm{~dB}$ is readily feasible. Furthermore, by having the signal and pump beam paths substantially equal makes the system insensitive to phase fluctuations and relaxes the requirements on phase noise and the coherence length of the laser source.

Single-frequency detection laser: The detection laser is preferably operated on a single-frequency mode (one longitudinal and one transverse mode). Operating the laser in the multimode regime results in a smaller coherence length, which can be overcome with the TWMPD by making the pump and signal beampaths smaller than the coherence length. However, in practice, having to keep the beampath difference under 1 meter can be very difficult while inspecting contoured parts. More importantly, multimode lasers are noisier than single-frequency lasers due to the mode beatings that produce both intensity and phase fluctuations.

The single-frequency laser cavity design is based on two main features. First, the cavity should be as short as possible to make the longitudinal modes selection easier or at least possible. Second, the power inside the laser cavity should be as large as possible to reduce the laser spiking damping time constant and then get a quiet zone in the laser pulse substantially free of intensity fluctuations, and also to get as more power output as possible.

In theory, a homogeneous gain medium placed inside a linear cavity oscillates in one single longitudinal mode. In effect, even solid-state lasers are not perfectly homogeneous, and additional modes can occur due to spatial hole burning. Several methods are used to produce single-frequency operation. The most useful are based on the insertion of interferometric filters inside the cavity so that only one frequency is above laser threshold [7]. The most common method of obtaining single-frequency output is to insert an etalon inside the cavity [8]. However, the etalon has to be tilted away from normal incidence such that the laser oscillation due to feedback between the cavity mirrors and the etalon reflections is suppressed. This tilt degrades beam spatial profile and power inside the cavity as well as the etalon selectivity because the multiple reflections of the beam do not recover completely [9]. Three-mirror configurations like Fox-Smith interferometers are efficient because of the high tunability of the free spectral range, but suffer from difficulties to get the proper alignment [10]. Furthermore high finesse interferometers necessitate highly reflecting mirrors. A loss of $1 \%$ for each interferometer mirror involves a roundtrip overall loss of more than $10 \%$. This involves, along with less laser power, less stability.

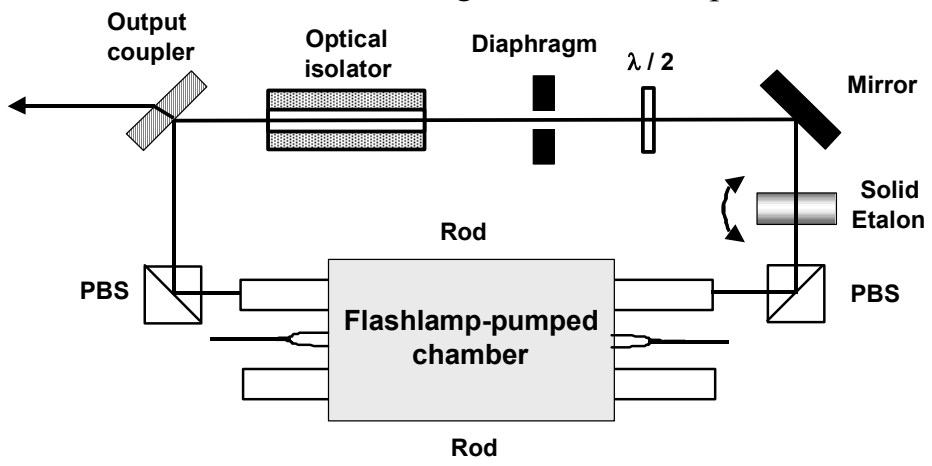

Figure 1. Setup of the laser cavity.

The laser oscillator we built is based on a unidirectional ring cavity. For several reasons this configuration is better than a linear cavity. As mentioned earlier, to make the selection of one longitudinal mode easier, the separation between adjacent longitudinal modes has to be as large as possible. The length corresponding to a round trip within 
the cavity must then be made as short as possible, this length being limited by the size of the components inside the cavity. Since in a ring cavity the elements are passed once instead of twice as in a linear cavity, ring cavities are for this reason advantageous [11]. Furthermore, the ring laser configuration avoids spatial hole burning since it is a travelling wave oscillator [12]. Figure 1 shows a schematic layout of the system. The Nd:YAG rod is mounted inside a dual-rod, single flashlamp-pumped chamber. The flashlamp controller is able to generate pulse durations from 50 to $300 \mu \mathrm{s}$, at repetition rates from 1 to $100 \mathrm{~Hz}$. The polarization selectivity is made by two polarizing beamsplitters also used as cavity mirrors. A half-wave plate coupled with a Faraday rotator prevents the clockwise oscillation of the laser. An iris was used to achieve TEM00 single-transverse-mode operation. The laser output modal content is continuously monitored by three devices. The Fourier transform of a signal acquired by a fast photodetector monitors the beating of adjacent longitudinal modes. The appearance of a frequency peak in the few hundred Megahertz frequency range means that two successive longitudinal modes are above lasing threshold. The beating of transverse modes in the few tens of Megahertz frequency range is also monitored by the same method. In addition, the ring patterns of an extra-cavity etalon are imaged on a CCD camera to monitor longitudinal modes separated by one or more spectral range of the intra-cavity etalon.

The laser cavity length is minimized, such that its free spectral range (FSR) is about $650 \mathrm{MHz}$. We observed that a loss of $5 \%$ between longitudinal modes is sufficient to yield a single-frequency regime. Therefore, an etalon with a transmission peak containing approximately ten longitudinal modes.

We operated the laser at $5 \mathrm{~Hz}$ repetition rate, with different pulse voltages of about $180 \mu$ s duration on the flashlamp. Figure 2 shows typical pulses obtained in the stable, single-frequency regime, which exhibits an intense spiking zone with a relaxation time close to $25 \mu \mathrm{s}$. The noise around $700 \mathrm{kHz}$ brought by these relaxation oscillations renders the laser useless for laser-ultrasonic experiments in the first $50 \mu \mathrm{s}$ of the pulse. The quiet zone is characterized by the absence of longitudinal and transverse modes cavity beat frequencies, which confirms single-frequency operation. In that zone, power fluctuations represent approximately $5 \%$ of the average power.
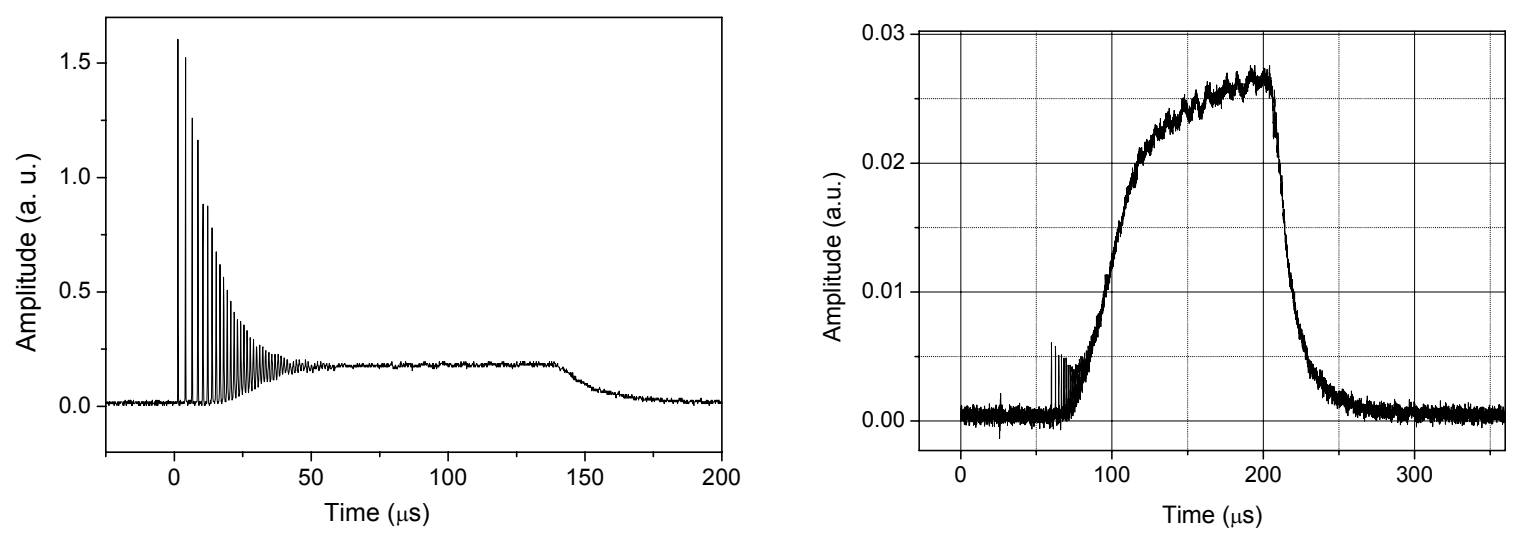

Figure 2. Temporal profile of an output laser pulse. Figure 3. Temporal profile of a two-pass amplified pulse.

We obtained a maximal output power of $35 \mathrm{~W}$ per pulse over a duration of $100 \mu \mathrm{s}$, which is typically two orders of magnitude larger than cw sources commonly used for laser-ultrasonic applications [13]. However, the investigations of low reflectivity or rough surface materials require sometimes higher power. In order to obtain more power, we used the second rod in the chamber as an amplifier. After a first pass, a maximal power of $400 \mathrm{~W}$ is obtained. Since it is less than the saturation intensity of Nd:YAG, it is possible to gain more energy with a second pass. After two passes we obtained a maximal gain of 25, leading to a power of $900 \mathrm{~W}$. Temporal profile of typical pulses with the highest gain is given in Fig. 3. Power fluctuations at the top of the injected and amplified pulses are both the same (about $5 \%$ of the total power), indicating that perturbations between the pumping processes in the oscillator and the amplification rods such as, for instance, optical leakage, in which photons in the first rod are scattered through the second rod and perturb the amplification gain, are negligible. It should be noted that the relaxation oscillations that appear at the beginning of the pulse have strongly reduced amplitude compared 
to the one in the initial oscillator pulse. This is explained by the delay occurring in the present set-up between the gain built-up and the damping of the oscillations.

Characteristics of the detection laser used with a two-wave mixing based photorefractive demodulator: Performance of our detection laser coupled to a TWMPD using a GaAs photorefractive crystal operating in the anisotropic diffusion configuration [5], i. e. without voltage applied to the crystal, is investigated. A polarizing beamsplitter separates the incident laser beam into the pump and signal beams. A phase modulator placed on the signal beampath is used to simulate the ultrasonic surface motion of the inspected part. In the actual configuration, the pump and signal beams propagate at an angle of $75^{\circ}$ outside the crystal from each other and have the same polarization. At the output of the crystal, a quarter-wave plate adds a quadrature between the local oscillator beam (or diffracted pump beam) and the signal beam. A half-wave plate and a PBS divide the whole power into two parts according to axes at $45^{\circ}$ from the propagation plane. Each part is focused on each detector of a balanced receiver in order to reduce intensity fluctuations by a factor of at least $30 \mathrm{~dB}$ over the $14 \mathrm{MHz}$ wide frequency bandwidth of the detectors [14]. As a result, phase modulations are transformed into intensity modulations. The best signal-tonoise ratio is obtained in the shot-noise regime for which the noise measured by the detector is proportional to the square root of the laser power. This means that in this regime the signal-to-noise ratio increases as the square root of the laser power.

The detection limit is defined as the minimum surface displacement (i.e., the displacement for which the SNR is equal to 1) that can be measured with the system for an electronic bandwidth of $1 \mathrm{~Hz}$ and a laser power of $1 \mathrm{~W}$. In the undepleted pump approximation, in which the signal power is negligible compared to the pump power, the detection limit and is given by [5]:

$$
\delta_{\min }=\sqrt{\frac{2 h v \Delta f}{\eta P_{0}}} \cdot \frac{\lambda}{4 \pi} \cdot \frac{\sqrt{1+\left(\frac{\Gamma d}{2}\right)^{2}}}{\Gamma d} \cdot \exp \left(\frac{\alpha d}{2}\right)
$$

where $\mathrm{h}$ is the Planck's constant, $\eta$ is the quantum efficiency of each detector $(0.93$ for InGaAs detectors used here), $\mathrm{P}_{0}$ is the laser power incident on the crystal, $v$ is the frequency of the laser, $\Delta \mathrm{f}$ is the detector bandwidth, $\alpha$ is the absorption coefficient, $\Gamma$ is the photorefractive gain, and $\mathrm{d}$ is the length of the crystal. For an incident signal power of $2.8 \mathrm{~mW}$ and a $14 \mathrm{MHz}$ detection bandwidth (from 1 to $15 \mathrm{MHz}$ ), the measured minimal detectable rms displacement dmin is $1.3 \times 10^{-2} \mathrm{~nm}$, which gives a detection limit of $1.85 \times 10^{-7} \mathrm{~nm}(\mathrm{~W} / \mathrm{Hz})^{1 / 2}$. The absorption coefficient and the photorefractive gain of the GaAs crystal are $1.43 \mathrm{~cm}^{-1}$ and $0.65 \mathrm{~cm}^{-1}$ respectively. The crystal has a length of $1 \mathrm{~cm}$. This results in a theoretical detection limit of $1.8 \times 10^{-7} \mathrm{~nm}(\mathrm{~W} / \mathrm{Hz})^{1 / 2}$, which is in good agreement with the experimental value.

Another important parameter of our detection laser is its coherence length, which is in general limited by the spectral bandwidth of the lasing mode. The coherence length does not affect the performance of the TWM photorefractive system as long as the pump and signal beampath difference can be made smaller than the coherence length. As a consequence, the beams that interfere in the crystal are always coherent and then write a hologram in the crystal, leading to an optimal demodulation. In practice, having to keep the beampath difference below 1 meter can be very difficult while inspecting contoured parts. By measuring the demodulation rms amplitude as a function of the delay between pump and signal, we obtained a coherence length of approximately $20 \mathrm{~m}$ for our laser, which is large enough for a practical system when this detection laser is coupled to a photorefractive demodulator.

The photorefractive grating buildup time must obviously be shorter than the detection laser pulse duration, otherwise no hologram is written in the crystal and the demodulator doesn't work. Moreover, a short buildup time reduces the sensitivity of the TWMPD to the longitudinal and transverse motion of the inspected sample [15]. On the other hand, the ultrasonic frequency response of the TWMPD is similar to a high pass RC filter and the grating buildup time affects the ultrasonic cut-off frequency. For example, a response time of $1 \mu$ s correspond to a $100 \mathrm{kHz}$ frequency cutoff [5]. A response time around $1 \mu \mathrm{s}$ is a good compromise between low ultrasonic frequency sensitivity and the rapidity of the TWMPD to adapt itself to environmental changes. The grating buildup time is reduced with the light power density impinging on the crystal. With the $35 \mathrm{~W}$ available from the detection laser, the grating buildup time of our TWMPD can be $1 \mu \mathrm{s}$ or shorter, as will be shown in the next section. It should also be noted that the high peak power spikes of the laser contributes to decrease the crystal buildup time. 
Compact and integrated laser-ultrasonic system: As stated in the introduction, a complete laser-ultrasonic system requires a generation laser, a detection laser and a phase demodulator. To build a compact and integrated laser-ultrasonic system we used one rod of the dual-rod pumping chamber for the detection laser and the other rod for the generation laser. Thus generation and detection units share the same pumping chamber, yielding a compact and practical tool for laser-ultrasonic applications. The generation laser cavity includes a Pockels cell to operate in the Q-switch regime. The laser delivers pulses of about $10 \mathrm{~ns}$ duration and $30 \mathrm{~mJ}$ of energy.

This compact laser-ultrasonic system was tested on an aluminum plate and on a steel tube. The setup for the tests is described in Fig. 4. The generation laser is focused on the sample in order to obtain high optical power density for ultrasound generation in ablation regime, thus giving rise to strong longitudinal wave emission perpendicular to the surface. The detection laser is collimated on the sample so that the detection spot is about $1 \mathrm{~mm}$. Light of the detection laser scattered by the sample is collected by a lens and sent to a multimode optical fiber ( $400 \mu \mathrm{m}$ core diameter, 0.22 numerical aperture). The fiber length is about 2 meters which is short enough so that the path difference between the pump and signal beams is smaller than the coherence length of the laser. The light collected back from the sample was brought by the optical fiber to the TWMPD. The first inspected sample is an aluminum plate with $6 \mathrm{~mm}$ thickness. The signal shown in Fig. 5 represents a typical single-shot measurement made in transmission mode (ultrasonic generation and detection spots on opposite sides of the sample). The first spike at several hundreds of nanoseconds comes from spurious reflections of the generation laser seen by the balanced receiver. This spike can be strongly reduced with a better optical isolation of the balanced receiver. A small lowfrequency noise is identified as residual laser intensity fluctuations caused by relaxation oscillations. This noise could be more attenuated with an improved attenuation factor of the balanced receiver, or by filtering out frequencies below $1 \mathrm{MHz}$. The measured time-of-flight between the first two longitudinal echoes is $2.08 \mu \mathrm{s}$, and the accurately measured thickness is $6.62 \mathrm{~mm}$, giving a longitudinal ultrasonic velocity of $6.36 \mathrm{~mm} / \mu \mathrm{s}$ in aluminum. Between the first and second echoes a spike related to shear waves is denoted.

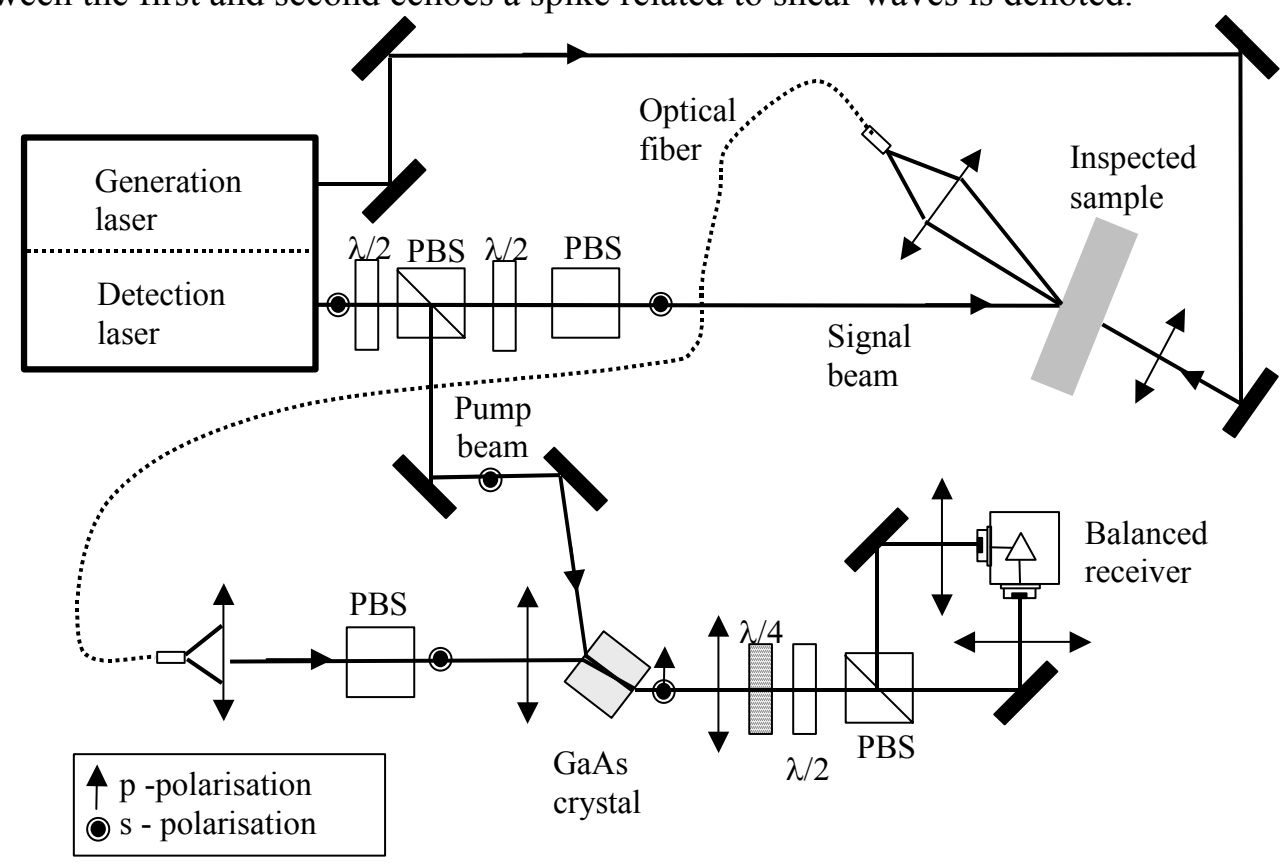

Figure 4. Experimental setup of laser-ultrasonic measurements on metallic sample.

Reflection measurements (ultrasonic generation and detection on the same side) were also carried out on a steel tube with $9.59 \mathrm{~mm}$ thickness. Since the two lasers are at the same wavelength, the generation and detection spots are separated by about $5 \mathrm{~mm}$ to limit the blindness of the balanced receiver by the short light pulse from the generation laser. Single-shot ultrasonic signal is reported on Fig. 6. The main difference here is the presence of a strong spike related to surface waves. The lower optical reflectivity of the steel tube contributes to a smaller signal- 
to-noise ratio of the longitudinal echoes when compared to the aluminum sample. Time-of-flight between two successive echoes is $3.14 \mu \mathrm{s}$, giving a measured longitudinal ultrasonic velocity in steel of $6.1 \mathrm{~mm} / \mu \mathrm{s}$. The compact integrated laser-ultrasonic system was then successfully tested on metallic samples in both transmission and reflection configurations.

Conclusion: We have reported the performances of a new, compact and simple laser-ultrasonic system. The main feature of this system is a long pulse, single-frequency detection laser oscillator. While noisier and less stable than the commonly used master $\mathrm{CW}$ oscillator power amplifier scheme, the new detection laser gives the same small surface displacement detection limit when coupled to a photorefractive two-wave mixing phase demodulator. Due to the balanced receiver scheme and the two-beam interferometer principle of this demodulator, the intensity and phase fluctuations of the laser can be strongly attenuated, relaxing the characteristics required from the detection laser. This demodulator also allows to operate with a detection laser

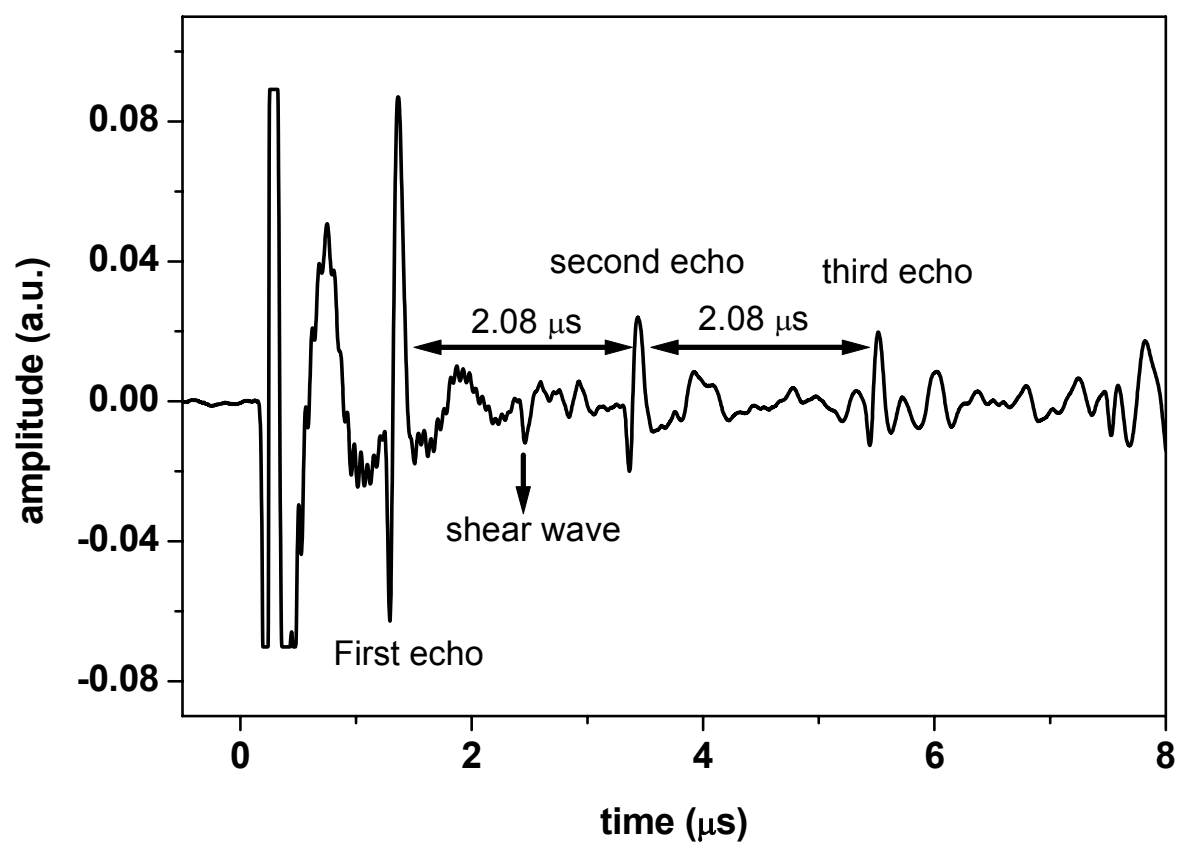

Figure 5. Typical laser-ultrasonic signal obtained on a $6 \mathrm{~mm}$ thickness aluminum sample with ultrasonic generation and detection spots on opposite sides of the sample. 


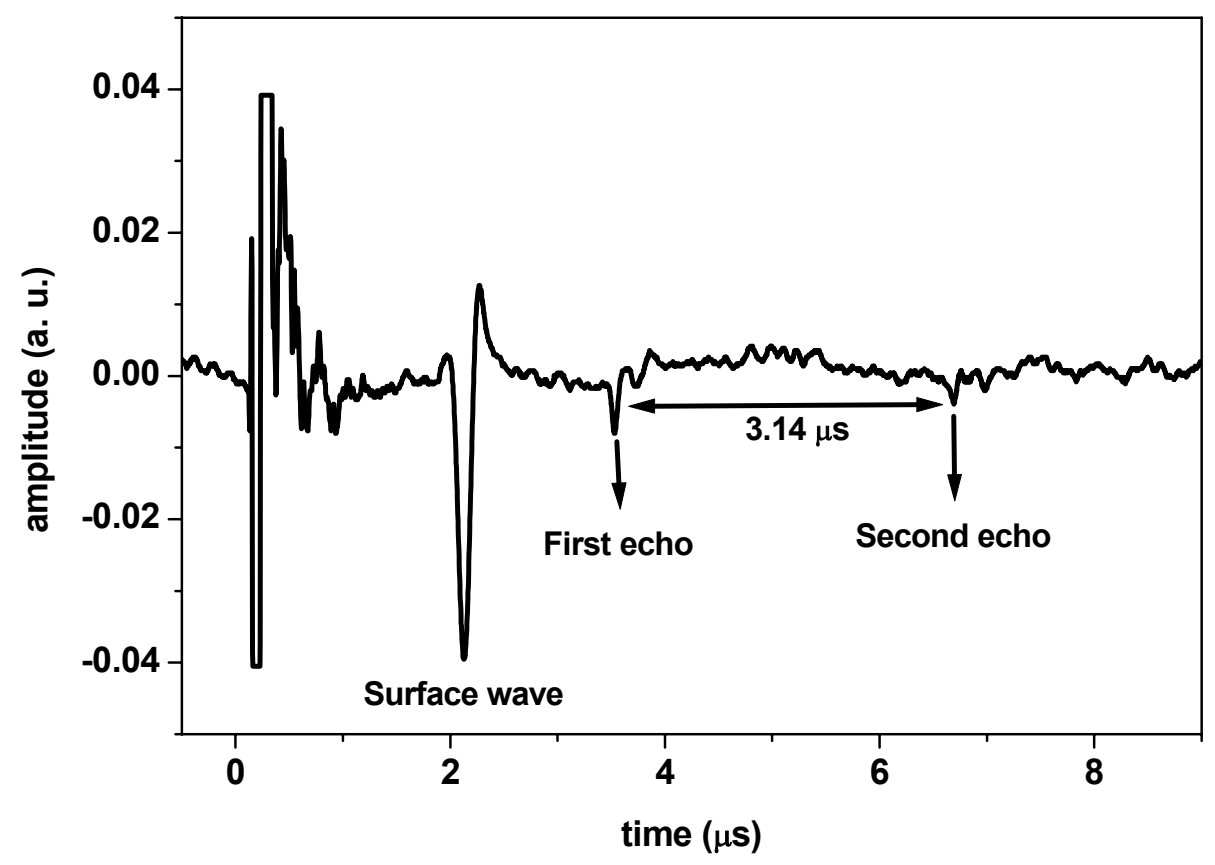

Figure 6. Typical laser-ultrasonic signal obtained on a $10 \mathrm{~mm}$ thickness steel sample with ultrasonic generation and detection on the same side of the sample.

having a very short coherence length. In practice, this coherence length cannot be made smaller than a few meters to allow the inspection of contoured parts. The laser delivers single-frequency pulses of $35 \mathrm{~W}$ power, which is typically two orders of magnitude larger than $\mathrm{cw}$ sources commonly used for laser-ultrasonic applications. Power of about $1 \mathrm{~kW}$ can be obtained when the second rod of a dual-rod pumping chamber is used as an amplifier. A compact laser-ultrasonic system is obtained when the second rod of the dual-rod pumping chamber is used to make a Q-switched Nd:YAG laser for ultrasonic generation. The complete system was succesfully tested on aluminium and steel parts. The tests were performed with generation and detection laser spots either on the same or on opposite sides of the part. This new design allows to build a compact and sensitive laser-ultrasonic system at an affordable cost.

\section{References:}

[1] C.B. Scruby and L.E. Drain, Laser-Ultrasonics: Techniques and applications, (Adam Hilger, Bristol, UK, 1990)

[2]. J.-P. Monchalin, Review of Progress in Quantitative Nondestructive Evaluation 12, 495 (1993).

[3] J.-P. Monchalin, IEEE Trans. Ultrason. Ferroelectrics and Freq.Cont. UFFC-33,485,1986.

[4] R. K. Ing and J. P. Monchalin, Appl. Phys. Lett. 59, 3233 (1991).

[5] A. Blouin and J. P. Monchalin, Appl. Phys. Lett. 65, 932 (1994).

[6] P. Delaye, A. Blouin, J.-P. Monchalin , G. Roosen, Trends in Optical Nondestructive testing and Inspection, Elsevier 2000, pp 519-532, P.K. Rastogi and D. Inaudi, Editors.

[7] P. W. Smith, Proc. IEEE 60, 422 (1972).

[8] S. A. Collins and G. R. White, Appl. Opt. 2, 448 (1963).

[9] W. R. Leeb, Appl. Phys. 6, 267 (1975).

[10] P. W. Smith, IEEE J. Quant. Electron. QE-2, 666 (1966).

[11] P. W. Smith, IEEE J. Quant. Electron. QE-4, 485 (1968).

[12] A. R. Clobes and M. J. Brienza, Appl. Phys. Lett. 21, 265 (1972).

[13] T. J. Kane and R. L. Byer, Opt. Lett. 10, 65 (1985). 
[14] B. Campagne, A. Blouin, L. Pujol, J. P. Monchalin, Rev. Sci. Instr. 72, 2478 (2001).

[15] B. Campagne, A. Blouin, C. Néron, J.-P. Monchalin, Review of Progress in Quantitative Nondestructive Evaluation, 22, 273, (2003). 